\title{
Spanning Trees of Different Weights
}

\author{
A. SCHRIJVER AND P. D. SEYMOUR
}

\begin{abstract}
Z. Füredi and D. Kleitman proved that if an integer weight is assigned to each edge of a complete graph on $p+1$ vertices, then there is a spanning tree whose edges have weights summing to zero modulo $p$. This result has a number of conjectured extensions; and in this paper we prove some of them when $p$ is prime. In particular, we prove that for any graph $G$ and prime $p$, if integer weights can be assigned to the edges of $G$ so that no spanning tree has weights summing to zero modulo $p$, then such a weighting can be chosen that is $(0,1)$-valued. We also prove that, under appropriate hypotheses, there are many spanning trees, all with different total weight modulo $p$. Matroid extensions of these last results generalize theorems from additive number theory.
\end{abstract}

\section{Introduction}

The following theorem is due to Z. Füredi and D. Kleitman [4].

THEOREM 1.1. Let $\Gamma$ be an abelian group of order $p$, and let $w: E\left(K_{p+1}\right) \rightarrow$ $\Gamma$ be a function. Then there is a spanning tree $T$ of $K_{p+1}$ such that $w(T)=0$. $\left(K_{n}\right.$ denotes the complete graph with $n$ vertices; $w(T)$ means $\sum_{e \in E(T)} w(e)$, where the summation is in $\Gamma$.)

This settled affirmatively a conjecture of Bialostocki and Dierker [1], who proved Theorem 1.1 for $p$ prime. Theorem 1.1 has given rise to several attempted generalizations. For instance, the second author proposed the following (see [4]).

CONJECTURE 1.2. Let $\Gamma$ be an abelian group of order $p$, let $G$ be a $p$ connected graph with $|V(G)| \equiv 1(\bmod p)$, and let $w: E(G) \rightarrow \Gamma$ be a function. Then there is a spanning tree $T$ of $G$ such that $w(T)=0$.

This is currently still open, even for $\Gamma$ cyclic. A stronger conjecture, as we shall show in Section 3, is the following.

1980 Mathematics Subject Classification (1985 Revision). Primary 05B35; Secondary 05C05, $10 \mathrm{~J} 15$.

This research was performed under a consulting agreement with Bellcore. 
CONJECTURE 1.3. Let $\Gamma$ be an abelian group of order $p$, let $G$ be a graph such that for every $A \subseteq E(G)$ there is a spanning tree $T$ of $G$ with $|A \cap E(T)| \equiv 0(\bmod p)$, and let $w: E(G) \rightarrow \Gamma$ be a function. Then there is a spanning tree $T$ of $G$ with $w(T)=0$.

Conjecture 1.3 would also imply

CONJECTURE 1.4. Let $p \geq 1$, and let $G$ be a graph. If there is a function $w: E(G) \rightarrow \mathbf{Z}_{p}$ such that $w(T) \neq 0$ for every spanning tree $T$, then there is such a function that is $(0,1)$-valued.

$\left(\mathbf{Z}_{p}\right.$ denotes the additive group of integers modulo $p$.)

Our objective in this paper is to prove Conjecture 1.3 (and hence Conjectures 1.2 and 1.4) when $p$ is prime. In fact, we think we can prove it when $p$ is a prime power or a product of two primes, but if those results are correct they will be published elsewhere. Sections 2 and 3 contain the proof of Conjecture 1.3, while in Section 4 we discuss a possible extension to the nonprime case.

Since all these questions are capable of formulation in matroid terms, and since all our proofs work just as well for matroids as for graphs, it seems natural to work in terms of matroids for greater generality and clarity. We assume a knowledge of elementary matroid theory; for an introduction and for all undefined terms, see [8].

\section{Bases of different weight}

If $M$ is a matroid, we denote its set of elements by $E(M)$, and the rank of a subset $X \subseteq E(M)$ by $\operatorname{rk}(X)$, or $\mathrm{rk}_{M}(X)$ in cases of ambiguity. We write $\operatorname{rk}(M)$ for $\operatorname{rk}(E(M))$. Let $M$ be a matroid, $\Gamma$ an abelian group, and $w$ : $E(M) \rightarrow \Gamma$ some function. For $g \in \Gamma$, we denote $\{e \in E(M): w(e)=g\}$ by $w^{-1}(g)$. For $X \subseteq E(M)$ we denote $\sum_{e \in X} w(e)$ by $w(X)$, and $w(M)$ denotes the set $\{w(B): B$ is a base of $M\}$. Our object in this section is to prove the following.

THEOREM 2.1. Let $p$ be prime, let $M$ be a matroid and let $w: E(M) \rightarrow$ $\mathbf{Z}_{p}$ be a function. Then

$$
|w(M)| \geq \min \left(p, 1-\operatorname{rk}(M)+\sum_{g \in \mathbf{Z}_{p}} \mathrm{rk}\left(w^{-1}(g)\right)\right) .
$$

To prove Theorem 2.1 we shall need the following result, the CauchyDavenport theorem (see [5]).

TheOREM 2.2. Let $p$ be prime, and let $A, B \subseteq \mathbf{Z}_{p}$ with $A, B \neq 0$. Then $|A+B| \geq \min (p,|A|+|B|-1)$.

$$
(A+B \text { denotes }\{a+b: a \in A, b \in B\} .)
$$

Proof of Theorem 2.1. We proceed by induction on $\operatorname{rk}(M)$. If $\operatorname{rk}(M)=$ 0 then the result is trivial, and so we may assume that $\operatorname{rk}(M) \geq 1$. Let 
$f \in E(M)$ with $\operatorname{rk}(\{f\})=1$. Let

$$
I=\left\{g \in \mathbf{Z}_{p}: \operatorname{rk}\left(w^{-1}(g) \cup\{f\}\right)=\operatorname{rk}\left(w^{-1}(g)\right)\right\} .
$$

(1) For every base $B$ of $M$ with $f \in B$, and for every $g \in I, w(B)+g-$ $w(f) \in w(M)$.

For this is trivial if $g=w(f)$, and so we assume that $g \neq w(f)$, that is, $f \notin w^{-1}(g)$. Since $\operatorname{rk}\left(w^{-1}(g) \cup\{f\}\right)=\mathrm{rk}\left(w^{-1}(g)\right)$ there is a circuit $C$ of $M$ with $f \in C \subseteq\{f\} \cup w^{-1}(g)$. Let $D$ be the cocircuit of $M$ with $D \cap B=\{f\}$. Since $C \cap D \neq\{f\}$ we may choose $e \neq f$ with $e \in C \cap D$. Since $e \in C-\{f\} \subseteq w^{-1}(g)$ it follows that $w(e)=g$; and since $e \in D$ it follows that $B^{\prime}=(B-\{f\}) \cup\{e\}$ is a base. But $w\left(B^{\prime}\right)=w(B)+w(e)-w(f)$, and the claim follows.

Let $N=\min \left(p, 1-\operatorname{rk}(M)+\sum_{g \in \mathbf{Z}_{p}} \operatorname{rk}\left(w^{-1}(g)\right)\right)$, and let $A=\{w(B): B$ is a base of $M$ and $f \in B\}$.

(2) $|A| \geq N-|I|+1$.

For let $M^{\prime}$ be the matroid obtained by contracting $f$; thus, $E\left(M^{\prime}\right)=$ $E(M)-\{f\}$, the bases of $M^{\prime}$ are all the sets $B-\{f\}$ where $B$ is a base of $M$ containing $f$, and its rank function is given by $\operatorname{rk}_{M^{\prime}}(X)=\operatorname{rk}_{M}(X \cup\{f\})-1$ for $X \subseteq E(M)-\{f\}$. Let $w^{\prime}$ be the restriction of $w$ to $E\left(M^{\prime}\right)$. Now for $g \in \mathbf{Z}_{p}$, if $g \neq w(f)$ then

$$
\begin{aligned}
\mathrm{rk}_{M^{\prime}}\left(w^{\prime-1}(g)\right) & =\mathrm{rk}_{M^{\prime}}\left(w^{-1}(g)\right) \\
& =\operatorname{rk}_{M}\left(w^{-1}(g) \cup\{f\}\right)-1 \\
& = \begin{cases}\operatorname{rk}_{M}\left(w^{-1}(g)\right) & \text { if } g \notin I, \\
\operatorname{rk}_{M}\left(w^{-1}(g)\right)-1 & \text { if } g \in I,\end{cases}
\end{aligned}
$$

while if $g=w(f)$ then

$$
\mathrm{rk}_{M^{\prime}}\left(w^{\prime-1}(g)\right)=\mathrm{rk}_{M^{\prime}}\left(w^{-1}(g)-\{f\}\right)=\mathrm{rk}_{M}\left(w^{-1}(g)\right)-1 .
$$

Thus

$$
\sum_{g \in \mathbf{Z}_{p}} \mathrm{rk}_{M^{\prime}}\left(w^{\prime-1}(g)\right)=\sum_{g \in \mathbf{Z}_{p}} \mathrm{rk}_{M}\left(w^{-1}(g)\right)-|I|,
$$

because $g \in I$ if $g=w(f)$. From the inductive hypothesis,

$$
\begin{aligned}
w^{\prime}\left(M^{\prime}\right) & \geq \min \left(p, 1-\operatorname{rk}\left(M^{\prime}\right)+\sum_{g \in \mathbf{Z}_{p}} \mathrm{rk}_{M^{\prime}}\left(w^{\prime-1}(g)\right)\right) \\
& =\min \left(p, 2-\operatorname{rk}(M)+\sum_{g \in \mathbf{Z}_{p}} \mathrm{rk}_{M}\left(w^{-1}(g)\right)-|I|\right) \\
& \geq N-|I|+1 .
\end{aligned}
$$

But $A=w^{\prime}\left(M^{\prime}\right)+\{w(f)\}$, and so $|A|=\left|w^{\prime}\left(M^{\prime}\right)\right|$. The claim follows. 
Let $C=\{g-w(f): g \in I\}$. By (1), $A+C \subseteq w(M)$. Now $A \neq \varnothing$ since $M^{\prime}$ has a base, and $C \neq \varnothing$ since $w(f) \in I$. By Theorem 2.2 and (2),

$|w(M)| \geq|A+C| \geq \min (p,|A|+|C|-1) \geq \min (p,(N-|I|+1)+|I|-1)=N$, as required.

We shall use Theorem 2.1 to prove our main result, but it has some other pretty consequences, which we shall discuss now before continuing the main proof. We remark first that Theorem 2.1 is a generalization of the CauchyDavenport theorem, Theorem 2.2. For given $A, B$ as in Theorem 2.2, let $M$ be the disjoint union of two uniform matroids of rank 1 , with $|A|$ and $|B|$ elements respectively, and define $w$ in the natural way; then Theorem 2.2 follows by applying Theorem 2.1 .

Theorem 2.1 also implies

THEOREM 2.3. Let $p$ be a prime, let $M$ be a matroid, and let $w: E(M) \rightarrow$ $\mathbf{Z}_{p}$ be such that for every cocircuit $D$ of $M$ there exist $e, f \in D$ with $w(e) \neq$ $w(f)$. Then $w(M) \geq \min (p, \operatorname{rk}(M)+1)$.

Proof. Let $M^{*}$ be the dual of $M$. For each $g \in \mathbf{Z}_{p}, w^{-1}(g)$ is independent in $M^{*}$, by assumption, and so

$$
\sum_{g \in \mathbf{Z}_{p}} \mathrm{rk}_{M^{*}}\left(w^{-1}(g)\right)=\sum_{g \in \mathbf{Z}_{p}}\left|w^{-1}(g)\right|=|E(M)| .
$$

By Theorem 2.1 applied to $M^{*}$,

$$
w(M)=w\left(M^{*}\right) \geq \min \left(p, 1-\operatorname{rk}\left(M^{*}\right)+|E(M)|\right)=\min (p, \operatorname{rk}(M)+1)
$$

as required.

We propose the following

Conjecture 2.4. Let $\Gamma$ be an abelian group of order $p$, and let $M$ be a matroid such that $\operatorname{rk}(M)$ is a multiple of $p$ and every cocircuit includes a base. Let $w: E(M) \rightarrow \Gamma$; then there is a base $B$ with $w(B)=0$.

Theorem 2.3 implies

THEOREM 2.5. If $p$ is prime then Conjecture 2.4 holds.

Proof. Let $\Gamma=\mathrm{Z}_{p}$. If some cocircuit $D$ of $M$ fails to satisfy the hypothesis of Theorem 2.3, then since it includes a base $B$ it follows that $w$ is constant on $B$, and since $p$ divides $|B|$ we deduce that $w(B)=0$. On the other hand, if there is no such cocircuit, then by Theorem 2.3, $w(M) \geq$ $\min (p, \operatorname{rk}(M)+1)$. Since we may assume $\operatorname{rk}(M) \neq 0$, and $p \operatorname{divides} \operatorname{rk}(M)$, it follows that $|w(M)|=p$, and so $w(M)=\mathbf{Z}_{p} \ni 0$, as required.

We observe that Theorem 2.5 implies the Bialostocki-Dierker theorem (Theorem 1.1 with $p$ prime), for every cocircuit of the polygon matroid of a complete graph includes a base. Conjecture 2.4 itself would imply Theorem 1.1 in full. The same situation occurs with the following theorem of Erdös, Ginzburg, and Ziv [3]. 
THEOREM 2.6. Let $p \geq 1$, and let $a_{1}, \ldots, a_{2 p-1} \in \mathbf{Z}_{p}$. Then some $p$ of them sum to zero.

This would be a consequence of Conjecture 2.4 by taking $M$ to be the uniform matroid of rank $p$ with $2 p-1$ elements. With $p$ prime, Theorem 2.6 is implied by Theorem 2.5. Incidentally, it was observed in [3] that the truth of Theorem 2.6 in general follows very easily from its truth in the prime case.

A further corollary of Theorem 2.1 is the following.

THEOREM 2.7. Let $p$ be prime, let $M$ be a matroid, and let $w: E(M) \rightarrow$ $\mathbf{Z}_{p}$ be a function. Suppose that $w(M) \neq \mathbf{Z}_{p}$. Then

$$
\sum_{g \in \mathrm{Z}_{p}} \mathrm{rk}\left(w^{-1}(g)\right)-\mathrm{rk}(M)<p-1
$$

Proof. By Theorem 2.1,

$$
\min \left(p, 1-\operatorname{rk}(M)+\sum_{g \in \mathbf{Z}_{p}} \operatorname{rk}\left(w^{-1}(g)\right)\right) \leq|w(M)|<p .
$$

The result follows.

\section{Bases of weight zero}

In this section we prove our main result, the following matroid generalization of Conjecture 1.3 for $p$ prime.

THEOREM 3.1. Let $p$ be prime, let $M$ be a matroid such that for all $A \subseteq$ $E(M)$ there is a base $B$ with $|A \cap B| \equiv 0(\bmod p)$, and let $w: E(M) \rightarrow \mathbf{Z}_{p}$ be a function. Then there is a base $B$ with $w(B)=0$.

We begin with the following, which is Rado's theorem [7] applied to the collection of sets consisting of $a_{i}$ copies of $A_{i}$ for each $i$.

THEOREM 3.2. Let $M$ be a matroid, and let $A_{i}(i \in J)$ be subsets of $E(M)$, mutually disjoint and with union $E(M)$. Let $a_{i}(i \in J)$ be nonnegative integers. Suppose that $\sum_{i \in I} a_{i} \leq \operatorname{rk}\left(\bigcup A_{i}: i \in I\right)$ for all $I \subseteq J$, and $\sum_{i \in J} a_{i}=\operatorname{rk}(M)$. Then there is $a$ base $B$ with $\left|B \cap A_{i}\right|=a_{i} \quad(i \in J)$.

We shall use Theorem 3.2 to prove the following.

THEOREM 3.3. Let $M$ be a matroid, and let $A_{i}(i \in J)$ be subsets of $E(M)$, mutually disjoint and with union $E(M)$. Let $p$ be an integer such that

$$
p>\max \left(\sum_{i \in J} \mathrm{rk}_{M}\left(A_{i}\right)-\mathrm{rk}(M), \sum_{i \in J} \mathrm{rk}_{M^{*}}\left(A_{i}\right)-\operatorname{rk}\left(M^{*}\right)\right) .
$$

Let $b_{i}(i \in J)$ be integers such that for all $I \subseteq J$ there is a base $B$ with

$$
\left|B \cap \bigcup_{i \in I} A_{i}\right| \equiv \sum_{i \in I} b_{i}(\bmod p) \text {. }
$$

Then there is a base $B$ such that for all $i \in J,\left|B \cap A_{i}\right| \equiv b_{i}(\bmod p)$. 
Proof. For each $i \in J$ there is a base $B$ with $\left|B \cap A_{i}\right| \equiv b_{i}(\bmod p)$. Let $a_{i}=\left|B \cap A_{i}\right|$. Then

(1) For each $i \in J, a_{i} \equiv b_{i}(\bmod p)$ and $\left|A_{i}\right|-\mathrm{rk}_{M^{*}}\left(A_{i}\right) \leq a_{i} \leq \operatorname{rk}_{M}\left(A_{i}\right)$.

For with $B$ as above, $a_{i}=\left|B \cap A_{i}\right| \leq \mathrm{rk}_{M}\left(A_{i}\right)$ since $B \cap A_{i}$ is independent in $M$, and similarly

$$
\left|A_{i}\right|-a_{i}=\left|A_{i} \cap(E(M)-B)\right| \leq \mathrm{rk}_{M^{*}}\left(A_{i}\right)
$$

since $E(M)-B$ is a base of $M^{*}$.

(2) For all $I \subseteq J, \sum_{i \in I} a_{i} \leq \mathrm{rk}_{M}\left(\bigcup A_{i}: i \in I\right)$.

For let $A=\bigcup\left(A_{i}: i \in I\right)$. By hypothesis, there is a base $B$ of $M$ with $|B \cap A| \equiv \sum_{i \in I} b_{i} \equiv \sum_{i \in I} a_{i}(\bmod p)$. Now

$$
\begin{aligned}
p+\mathrm{rk}(M) & >\sum_{i \in J} \mathrm{rk}_{M}\left(A_{i}\right)=\sum_{i \in I} \mathrm{rk}_{M}\left(A_{i}\right)+\sum_{i \in J-I} \mathrm{rk}_{M}\left(A_{i}\right) \\
& \geq \sum_{i \in I} a_{i}+|B-A|=\sum_{i \in I} a_{i}-|B \cap A|+\operatorname{rk}(M),
\end{aligned}
$$

and so $|B \cap A|>\sum_{i \in I} a_{i}-p$. Since $|B \cap A| \equiv \sum_{i \in I} a_{i}(\bmod p)$ it follows that $|B \cap A| \geq \sum_{i \in I} a_{i}$ and hence $\operatorname{rk}_{M}(A) \geq \sum_{i \in I} a_{i}$, as required.

(3) $\sum_{i \in J} a_{i}=\mathrm{rk}(M)$.

For $\sum_{i \in J} a_{i} \leq \operatorname{rk}(M)$ (by setting $I=J$ in (2)). To prove the reverse inequality we observe that, by (1),

$$
\sum_{i \in J} a_{i} \geq \sum_{i \in J}\left|A_{i}\right|-\sum_{i \in J} \mathrm{rk}_{M^{*}}\left(A_{i}\right)>|E(M)|-\operatorname{rk}\left(M^{*}\right)-p=\operatorname{rk}(M)-p .
$$

But $\sum_{i \in J} a_{i} \equiv \sum_{i \in J} b_{i} \equiv \operatorname{rk}(M)(\bmod p)$, by setting $I=J$ in the hypothesis. Hence $\sum_{i \in J} a_{i} \geq \operatorname{rk}(M)$, and the claim follows.

From (2), (3), and Theorem 3.2, there is a base $B$ such that $\left|B \cap A_{i}\right|=$ $a_{i} \equiv b_{i}(\bmod p)$ for all $i \in J$, as required.

PROOF OF THEOREM 3.1. If $\sum_{g \in \mathbf{Z}_{p}} \mathrm{rk}_{M}\left(w^{-1}(g)\right)-\operatorname{rk}(M) \geq p-1$, then by Theorem 2.7 applied to $M, w(M)=\mathbf{Z}_{p} \ni 0$ as required. Likewise, if $\sum_{g \in \mathrm{Z}_{p}} \mathrm{rk}_{M^{*}}\left(w^{-1}(g)\right)-\mathrm{rk}\left(M^{*}\right) \geq p-1$, then by Theorem 2.7 applied to $M^{*}$, $w\left(M^{*}\right)=\mathbf{Z}_{p}$ and hence $w(M)=\mathbf{Z}_{p} \ni 0$, as required. We may assume then that both these inequalities fail to hold. By Theorem 3.3 (taking $J=Z_{p}$, and $A_{g}=w^{-1}(g)$ and $b_{g}=0$ for all $\left.g \in J\right)$ there is a base $B$ of $M$ such that $\left|B \cap w^{-1}(g)\right| \equiv 0(\bmod p)$ for all $g \in \mathbf{Z}_{p}$, and hence $w(B)=0$, as required.

The following reformulation of Theorem 3.1 is a matroid generalization of Conjecture 1.4 when $p$ is prime.

THEOREM 3.4. Let $p$ be a prime, and let $M$ be a matroid. If there is a function $w: E(M) \rightarrow \mathbf{Z}_{p}$ with $0 \notin w(M)$, then such a function may be chosen $(0,1)$-valued. 
Proof. By Theorem 3.1 there exists $A \subseteq E(M)$ such that $|A \cap B| \not \equiv$ $0(\bmod p)$ for every base $B$. Let $w(e)=1 \quad(e \in A), w(e)=0(e \notin A)$; then $0 \notin w(M)$.

Now we turn to the proof of Conjecture 1.2 when $p$ is prime. Let us say a matroid $M$ is p-connected if

$$
\mathrm{rk}(A)+\operatorname{rk}\left(A^{\prime}\right) \geq \operatorname{rk}(M)+p-1
$$

for every partition $\left(A, A^{\prime}\right)$ of $E(M)$ such that $\operatorname{rk}(A), \operatorname{rk}\left(A^{\prime}\right)<\operatorname{rk}(M)$. We require the following lemma.

LEMMA 3.5. Let $p \geq 1$, let $M$ be a p-connected matroid such that $\operatorname{rk}(M)$ $\equiv 0(\bmod p)$, and let $A \subseteq E(M)$. Then there is a base $B$ with $|B \cap A|$ $\equiv 0(\bmod p)$.

Proof. Let $A^{\prime}=E(M)-A$. If $A$ or $A^{\prime}$ includes a base $B$, then $|B \cap A| \equiv 0(\bmod p)$ since $|B|=\mathrm{rk}(M) \equiv 0(\bmod p)$, as required. We assume that $\operatorname{rk}(A), \operatorname{rk}\left(A^{\prime}\right)<\operatorname{rk}(M)$. Since $M$ is $p$-connected, $\operatorname{rk}(A)+\operatorname{rk}\left(A^{\prime}\right) \geq$ $\mathrm{rk}(M)+p-1$. By extending to a base of $M$ some maximal independent subset of $A^{\prime}$, we obtain a base $B_{1}$ with $\left|B_{1} \cap A^{\prime}\right|=\operatorname{rk}\left(A^{\prime}\right)$. Since

$$
\left|B_{1} \cap A\right|=\left|B_{1}\right|-\left|B_{1} \cap A^{\prime}\right|=\operatorname{rk}(M)-\operatorname{rk}\left(A^{\prime}\right) \leq \mathrm{rk}(A)-p+1,
$$

we may choose an independent subset $X \subseteq A$ with $B_{1} \cap A \subseteq X$ such that $|X| \equiv 0(\bmod p)$. Let $B$ be a base with $X \subseteq B \subseteq X \cup B_{1}$. Since $B_{1} \cap A \subseteq X$ it follows that $B \cap A=X$, and hence $|B \cap A| \equiv 0(\bmod p)$, as required.

From Theorem 3.1 and Lemma 3.5 we deduce the following.

THEOREM 3.6. Let $p$ be prime, let $M$ be a p-connected matroid such that $\operatorname{rk}(M) \equiv 0(\bmod p)$, and let $w: E(M) \rightarrow \mathbf{Z}_{p}$ be a function. Then there is a base $B$ with $w(B)=0$.

Theorem 3.6 implies that Conjecture 1.2 holds when $p$ is prime. For if $G$ is a $p$-connected graph, then its polygon matroid is a $p$-connected matroid (see [2]).

\section{The nonprime case}

So far, none of our results implies Theorem 1.1, because our results are only for $p$ prime, while Theorem 1.1 holds for $p$ composite as well. The main difficulty in handling the nonprime case is that the Cauchy-Davenport theorem does not hold. However, there is a form of the latter for the nonprime case, and even for general abelian groups, due to Kneser [6], as follows.

THEOREM 4.1. Let $\Gamma$ be an abelian group and let $A, B \subseteq \Gamma$ be finite. Then either $|A+B| \geq|A|+|B|-1$, or there exists a nonzero $g \in \Gamma$ such that $A+B+\{g\}=A+B$.

We would like to propose a conjecture generalizing Theorem 4.1 in the same way that Theorem 2.1 generalizes Theorem 2.2, the following. 
CONJECTURE 4.2. Let $\Gamma$ be an abelian group, let $M$ be a matroid, and let $w: E(M) \rightarrow \Gamma$ be a function. Then either

$$
|w(M)| \geq 1-\operatorname{rk}(M)+\sum_{g \in \Gamma} \mathrm{rk}\left(w^{-1}(g)\right)
$$

or there exists a nonzero $g \in \Gamma$ such that $w(M)+\{g\}=w(M)$.

If true, this would imply all the other conjectures in this paper. In particular, it would imply a "nonprime" form of Theorem 2.7, that given $\Gamma, M, w$ as in Conjecture 4.2, if

$$
1-\mathrm{rk}(M)+\sum_{g \in \Gamma} \mathrm{rk}\left(w^{-1}(g)\right)>|\Gamma|
$$

then there is a nonzero $g \in \Gamma$ such that $w(M)+\{g\}=w(M)$. That, in turn, combined with Theorem 3.3, would imply an extension of Theorem 3.1: that given $\Gamma, M, w$ as usual, if $0 \notin w(M)$ then there exists $A \subseteq E(M)$ such that $|B \cap A| \not \equiv 0(\bmod |\Gamma|)$ for all bases $B$ of $M$. That, together with Lemma 3.5, would imply a nonprime version of Theorem 3.6; the latter would imply Conjecture 2.4, and that would imply Theorems 1.1 and 2.6. Thus, we would very much like to prove Conjecture 4.2 . We can prove it if $\operatorname{rk}(M) \leq 2$ (even that is nontrivial, for it already contains Theorem 4.1), and we have convinced ourselves that it is true when $|\Gamma|$ is a power of a prime, or the product of two primes. But this last, if it is correct, will appear in a later paper.

\section{REFERENCES}

1. A. Bialostocki and P. Dierker, Zero sum Ramsey theorems, manuscript.

2. W. Cunningham, On matroid connectivity, J. Combin. Theory Ser. B 30 (1981), 94-99.

3. P. Erdös, A. Ginzburg, and A. Ziv, Theorem in the additive number theory, Bulletin Research Council of Israel 10 (1961), 41-43.

4. Z. Füredi and D. J. Kleitman, On zero-trees, manuscript.

5. H. Halberstam and K. F. Roth, Sequences, Vol. 1, Oxford University Press, 1966.

6. M. Kneser, Abschätzungen der asymptotischen Dichte von Summenmengen, Math. Zeit. 58 (1953), 459-484.

7. R. Rado, A theorem on independence relations, Quart J. Math. Oxford Ser. (2) 13 (1942), 83-89.

8. D. J. A. Welsh, Matroid theory, Academic Press, New York, 1976.

Mathematical Centre, Amsterdam

BELLCORE, NEW JERSEY 